\title{
Effect of Be-disk evolution on the global one-armed oscillations
}

\author{
Finny Oktariani ${ }^{1}$ and Atsuo T. Okazaki ${ }^{2}$ \\ ${ }^{1}$ Department of Cosmoscience, Graduate School of Science, Hokkaido University, Kita-ku, \\ Sapporo 060-0810, Japan \\ email: finny@astro1.sci.hokudai.ac.jp \\ ${ }^{2}$ Faculty of Engineering, Hokkai-Gakuen University, Toyohira-ku, Sapporo 062-8605, Japan \\ email: okazaki@elsa.hokkai-s-u.ac.jp
}

\begin{abstract}
We consider the effect of density distribution evolution on the global one-armed oscillation modes in disks around Be stars. Previous studies of global oscillations in Be disks assumed a power-law density distribution of the disk. However, observational results show that some Be stars exhibit evidence of formation and dissipation of the equatorial disk. This causes the disk density distribution can be far from a power-law form. Performing calculations for several times in the disk formation and dissipation stages, we find one-armed modes confined to the inner part of the disk in both stages. In the disk formation stage, the oscillation frequency stays approximately constant after the disk is fully developed. In the dissipation stage of the Be disk, the local precession frequency is, in general, higher than in the disk formation stage. Thus, we expect that $\mathrm{V} / \mathrm{R}$ periods become shorter as the innermost part of the disk starts to accrete.
\end{abstract}

Keywords. stars: emission-line, Be, stars: oscillations

\section{Introduction}

Be stars are non-supergiant B-type stars that show emission lines in their Balmer lines of hydrogen. The emission lines are attributed to a cool circumstellar disk around the star. Many Be stars show long-term $\mathrm{V} / \mathrm{R}$ variations which are variations of Violet $(\mathrm{V})$ to Red $(\mathrm{R})$ peak ratio in double peaked emission line profiles. This variation is attributed to the precession of global one-armed oscillation in Be disk (Okazaki 1991; Papaloizou et al. 1992). Some Be stars show evidences of formation and dissipation of disk, during which the density distribution in the disk is likely far from the usually-assumed, powerlaw distribution. We study the effect of the Be disk evolution on the global one-armed oscillation.

\section{Density distribution}

We calculated the density evolution in an isothermal, decretion disk around a $\mathrm{Be}$ star, solving a 1-D, diffusion-type equation of the surface density (e.g., equation (12) of Okazaki et al. 2002) for the formation and dissipation stages. The obtained results can be seen in the upper panels of Fig. 1. In the early times of the formation stage, we have a density distribution with steep slope. The mass is concentrated in the area near the star. Then, by the means of viscosity the mass flows to the outer part, making the density distribution closer to that with a power-law. In the dissipation stage, when no mass is ejected from the star, the disk is lost from the innermost part, causing a gap between the star and the disk. Then, the density distribution has a peak near the inner radius. We then calculate one-armed eigenmodes using the obtained density distribution at a particular time. 

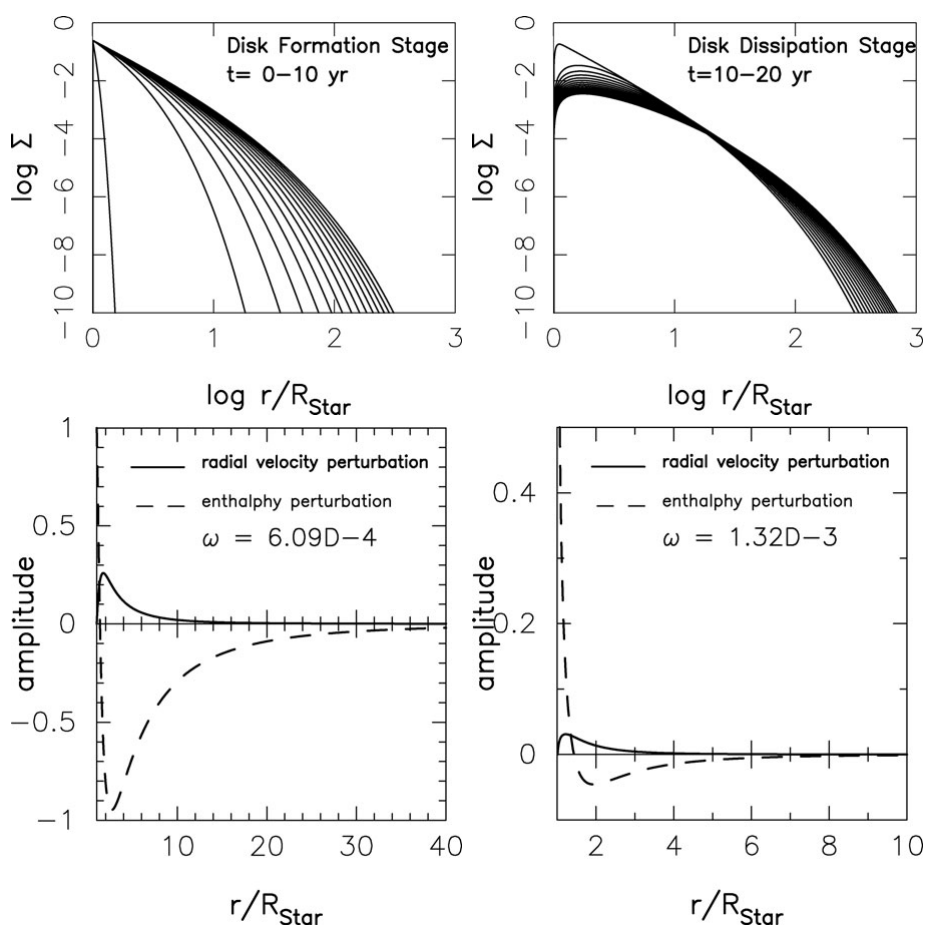

Figure 1. Surface density evolution in the disk around a single Be star in the formation stage (upper left panel) and dissipation stage (upper right panel). The two adjacent lines correspond to $t \approx 10$ months. We used viscosity parameter $\alpha=0.1$, and mass ejection rate $\dot{M} \sim 10^{-9} M_{\odot} \mathrm{yr}^{-1}$ in the disk formation stage. The lower panels show the eigenfunctions of the $m=1$ modes for a single Be star in the disk formation stage (lower left panel) and dissipation stage (lower right panel).

\section{Effect of disk density evolution on global one-armed modes}

We superposed linear global one-armed modes $\propto \exp [i(\omega t-\varphi)]$ in the unperturbed disk model. We found one armed modes confined to the inner part of the disk both in formation and dissipation stages. In the disk formation stage, $\omega$ stays approximately constant after the disk is fully developed. No eigenfrequency found before the disk is developed. In the disk dissipation stage, we found that the eigenmodes are more strongly confined than those in the formation stage, and $\omega$ also stays approximately constant throughout the stage. In the dissipation stage, the local precession frequency is generally higher than in the formation stage. Hence, we have higher $\omega$, i.e., a shorter period of $\mathrm{V} / \mathrm{R}$ variability. Comparing our results with those calculated using a power-law density distribution (Oktariani \& Okazaki 2009), we found that several years after the disk formation started, the eigenfrequency becomes close to the previous results. This is because the density distribution becomes close to the power-law form when the disk is fully developed.

\section{References}

Okazaki, A. T. 1991, PASJ, 43, 75

Okazaki, A. T., Bate, M. R., Ogilvie, G. I., \& Pringle, J. E. 2002, MNRAS, 337, 967

Oktariani, F. \& Okazaki, A. T. 2009, PASJ, 61, 57

Papaloizou, J. C., Savonije, G. J., \& Henrichs, H. F. 1992, A\&SA, 265, L45 